\title{
Current Strategies for Sustaining Drug Release from Electrospun Nanofibers
}

\author{
Shih-Feng Chou ${ }^{1}$, Daniel Carson ${ }^{1}$, Kim A. Woodrow ${ }^{1, \dagger}$ \\ Author Affiliation: \\ ${ }^{1}$ Department of Bioengineering, University of Washington \\ $372015^{\text {th }}$ Ave NE, Seattle WA, 98195-5061, USA \\ ${ }^{\dagger}$ Corresponding author: \\ Dr. Kim A. Woodrow \\ Foege N410D, Department of Bioengineering, University of Washington \\ $372015^{\text {th }}$ Ave NE, Seattle WA, 98195-5061, USA \\ 1-(206)-685-6831 \\ woodrow@uw.edu
}

\begin{abstract}
Electrospun drug-eluting fibers are emerging as a novel dosage form for multipurpose prevention against sexually transmitted infections, including HIV, and unintended pregnancy. Previous work from our lab and others show the versatility of this platform to deliver large doses of physico-chemically diverse agents. However, there is still an unmet need to develop practical fiber formulations for water-soluble small molecule drugs needed at high dosing due to intrinsic low potency or desire for sustained prevention. To date, most sustained release fibers have been restricted to the delivery of biologics or hydrophobic small molecules at low drug loading of typically $<1 \mathrm{wt} \%$, which is often impractical for most clinical applications. For hydrophilic small molecule drugs, their high aqueous solubility and poor partitioning and incompatibility with insoluble polymers makes long-term release even more challenging. Here we investigate several existing strategies to sustain release of hydrophilic small molecule drugs that are highly-loaded in electrospun fibers. In particular, we investigate what is known aboutthe design constraints required to realize multi-day release from fibers fabricated from uniaxial and coaxial electrospinning.
\end{abstract}

Keywords: electrospun fibers, uniaxial, coaxial, sustained release, drug delivery 


\section{Content}

1. Introduction

2. Material composition for sustained drug release from electrospun fibers

2.1. Polymers and excipients

2.3. Hydrophilic agents for sustained release

2.2. Analytical methods to characterize drug-polymer fiber dispersions

3. Uniaxial fiber design considerations for sustained release

3.1. Uniaxial electrospinning process and solution properties

3.2. Effect of polymer and drug dispersion on sustained release

4. Coaxial fiber design considerations for sustained release

4.1. Core-shell fiber electrospinning process and solution properties

4.2. Effect of core-shell composition and architecture on sustained release

4.2.1. Integrity of the core-shell structure

4.2.2. Shell thickness and composition

5. Conclusions and future perspectives 


\section{Introduction}

Electrospinning is an establishedtechnique to producesmall diameter fibers in the range of nanometers to several micrometers. Since the adventof using electrostatic force to produce fibers inthe 1930s [1],the processof electrospinning has become widely employed in many engineering fields including filtration [2-4], masking/fabrics [5-9], sensors [10-11], and energy-related applications [12]. The theory of electrospinning, which includes the role of processing variable, solution variables, architecture and mechanical properties, has been extensively reviewed by others in the past[13-15]. In addition, there have been several comprehensive reviews on scale-up processes for electrospinning and their applications in tissue engineering and drug delivery [1624].Amongthe biomedical applications, drug delivery isone of the most promising areas to employ electrospun materials. The advantages of using electrospun fibers in drug delivery include: (1) high drug loading (up to 60\%) and encapsulation efficiency (up to $100 \%$ ) [25-27], (2) polymer diversity to accommodatecompatibilitywith physicochemicallydistinct agents [28,29], (3) ability to modulate release[30-31], and (4) processsimplicity and cost-effectiveness [32].

Due to the platform versatility of electrospinning, there is an emerging interest to understand the role of electrospun materials in addressing clinical gaps associated with treatment and prevention of bacterial and viral infections. The development of longacting drug formulations is being sought in many of these applications to overcome challenges with adherence and emerging drug resistance. However, many antimicrobial agents require high and frequent dosing for efficacy due to theirlow intrinsic potency and short half-lives, when administered in vivo[33].For example, common antibiotics used to treat bacterial infections are typically dosed ata minimum of hundreds of milligrams per day for several weeks. Long-acting strategies for HIV prevention and treatment require similar dosing regimens forexisting antiretroviral drugs currently on the market [34].The high aqueous solubility of many lead antimicrobial agents further precludes their formulation into long-acting dosage forms by standard techniques such as milling to produce nano-sized crystalline drugs.A better understanding of the current design space required to achieve sustained release from electrospun fibers will helpinform the platform's applicability to address these specific clinical gaps in drug delivery.

The purpose of this review is to highlight current strategies using electrospun fibers for sustained drug release, particularly in applications where high drug dosing may be needed for clinical efficacy. We give specific attention to approaches for sustaining the release of hydrophilic small molecule drugs that are highly loaded in fiber formulations.In particular, we focus on examples of drug-eluting fibers that meet a minimum of $20 w t \%$ loading and achieve at least daily release of 10-100 milligrams of drugs for up to a week or longer.Much of the research and development in the field has employed uniaxial fibers or core-shell fibers fabricated by coaxial electrospinning (Table 1), which will be the main emphasis of this review. These studies highlightour current knowledge of thematerial compositions and solid-state characteristics needed to achievehigh dosing and sustained release of agents from electrospun fibers in applications requiringlong-acting clinical efficacy.

\section{Material composition for sustained drug release from electrospun fibers}




\subsection{Polymers and excipients}

The choice of polymer represents a key component in the development of sustained release electrospun fibers (Table 1). The seemingly unlimited number of natural and synthetic polymers that can be electrospun highlights the versatility of this platform. Therefore, the intended application of sustained release fibers often dictates polymer choice. Both non-biodegradable and biodegradable polymers have been used for sustained release from electrospun fibers $[35,36]$. The most prevalent polymers used in electrospun fibers for sustained release are biodegradable polyesters such as PLA, polyglycolic acid (PGA), poly(lactic-co-glycolic) acid (PLGA), and polycaprolactone $(\mathrm{PCL})$ [37]. Common non-degradable synthetic polymers employed for sustained drug release included polyurethane (PU), polycarbonate, and nylon-6.Verreck et al. [36] demonstrated controlled release of two hydrophobic drugs loaded at 10-40 wt\% in PU electrospun fibers. While some naturally occurring polymers such assilk, collagen, gelatin, alginate, and chitosan have been investigated for sustained drug release from fibers, their shear-thinning properties make them challenging to electrospin alone or at high yield. These polymers have been reviewed by Piskin et al. [38] on their ability to electrospin into nanofibers.

The mechanism of drug release from fibers withthese polymeric compositions can be attributed to a combination ofdiffusive processes, polymer degradation, drug partitioning in polymers, and drug dissolution. In both degradable and non-degradable polymers that are also non-swellable, drugs must diffuse through the solid polymer matrix before diffusion into the bulk. The rate of drug diffusion out of the polymer matrix reflects a number of processes including the rate of water diffusion intothe polymer, partitioning and solubility of the drug between the polymer and the bulk, and diffusivity of the drug in the polymer.For non-degradable polymers, the average distance a drug diffusesthrough the polymer matrix is dependent on a fixed geometry. In contrast, the geometry for a biodegradable polymer changes with time and the average distance of diffusion will vary depending on the rate of polymer degradation.

Semi-crystalline and glassy polymers are often used for sustained drug release due to the slower rate of water diffusion into these materials. Cross-linked polymers can also impede water penetration, which make them useful materials for some sustained release applications [39]. Yohe et al.[40]demonstrated thatcontrolling the hydrophobicity of PCL fibers with a dopant is an effective strategy to tune drug release by affecting the rate of water diffusion into the electrospun porous fiber network. In contrast, other excipients such as glycerol have been used to enhance the wetting process and accelerate drug release [25].Drug properties and form, in the final solid dispersion with the polymer fiber,will also greatly impact its observed release kinetics. As will be discussed in detail below, solvent compatibility with drug and solvent evaporation rate during the electrospinning process can be selected to impact the final solid state. In general, the electrospinning platform allows for a number of interconnected factors to be controlled but care must be taken to achieve designs for sustained drug release.

\subsection{Hydrophilic agents for sustained release}

Although a wide variety of agents can be incorporated into electrospun fibers [41], most examples of sustained drug release out to at least 7 days have been primarily limited to hydrophobic small molecule drugs or large biological macromolecules. These 
agents are more amenable to sustained release due to their poor solubility, large size, or preferential partitioning into insoluble polymers. In contrast, hydrophilic small molecule drugs represent a major challenge in sustained release because of high solubility with release media, poor partitioning, and low compatibility with many hydrophobic polymers. Drug-polymer compatibility correlates strongly with the ability to fully encapsulate drugs and accomplish sustained release. Hydrophilic small molecules tend to have low solubility with hydrophobic solvent-polymer systems. In these situations, the hydrophilic molecules are inherently more likely to partition to the surface of fibers.

A majority of the studies investigating hydrophilic small molecule drug loading and release from electrospun fibers have focused on antibiotics and some antiviral compounds (Table 1). The physico-chemical diversity of small molecule drugs with respect to parameters such as aqueous solubility, partition coefficient, ionization and pKa, molecular dipole, glass transition and melt temperature, are all important factors that will contribute to its interaction with the solvent and polymer both in solution and in the final solid dispersion. As such, using model hydrophilic compounds to extrapolate structure-function relationships between the fiber formulation characteristics and drug release kinetics should be interpreted with caution. For example, a recent study by Carson et al. [42] demonstrated the ability to tune the release 10-40 wt\% tenofovir from 24 hours out to 30 days, using PCL/PLGA electrospun fibers. The study showed that higher PCL or PLGA content yielded faster or slower diffusional release of tenofovir, respectively. The goal was to generalize the PCL/PLGA electrospun fiber platform to other hydrophilic small molecule drugs. However, the release of azidothymidine, maraviroc, raltegravir, and tenofovir disoproxil fumarate was much faster compared to tenovir, using equivalent PCL/PLGA fiber formulations. This suggests that even slight differences within the chemical structures of these compounds, compared to tenofovir, can affect rate of release. It appears that the presence of a phosphonic acid functional group on tenofovir creates a unique interaction with PCL/PLGA polymer backbones and inhibits release. Therefore, a deeper understanding of the drug-polymer-solvent interactions at all stages of the electrospinning process will require availability and access to lead clinical compounds.

The large drug loading needed for clinical applications that require high daily dosing (10-100 mg/dose) presents additional challenges for sustaining drug release from fibers. Higher drug loadingoften results in increased burst release of drug due to larger amounts of surface-associated drug and high surface area of fibers. In fact, sustained release of small molecule drugs from fibers has been typically performed with low drug loading $(<1 \mathrm{wt} \%)$, which limits clinical applications for treating or preventing many bacterial and viral infections. For example, Ball et al. [28] have fabricated nanofibers using various biodegradable polymers for sustained release. In theirstudies, several microbicides such as tenofovir, dapivirine, maraviroc, azidothymidine, acyclovir, glycerylmonolaurate were successfully incorporated into the nanofibers without any toxicity to various cells and explants. These nanofibers sustained release of the antiviral drugsbut were loaded at only $1 \mathrm{wt} \%$, which is not clinically relevant for the proposed applications [28]. In a separate example for the same clinical purpose, Huang et al. [43] loaded tenofovir disoproxil fumarate, a water-soluble antiretroviral prodrug, into fibers electrospun from a polymer that would undergo dissolution in response to a $\mathrm{pH}$ change 
induced by semen. However, they did not demonstrate sustained release of the prodrug or provide any analytical data describing the solid drug dispersion in the finished fibers.

\subsection{Analytical methods to characterize drug-polymer fiber dispersions}

Analytical techniques can be employed at different stages of the electrospinning process to investigate the structure-function relationship between formulation properties and performance attributes of the final drug-loaded polymer fibers. Solution properties of the solvent containing drug, polymer, and other excipients impact both the electrospinning process and resulting fibers. A critical solution viscosity is needed to maintainmolecular chain entanglement to form fibers during the electrospinning process and is measured with a rheometer. Although the solution viscosity will depend on the polymer and other factors,typical values of viscosity are reported in the range of 0.5 and 2 Pa's $[44,45]$. Drug-polymer interactions in solution can also induce a change in fluid surface tension, which affect Taylor cone formation and jet initiation. A drug-polymer solution with low surface tension, achieved by using nonpolar or weakly polar solvents, favors the formation of a Taylor cone at a much lower electrospinning voltage [46]. A tensiometer is used to measure surface tension. Furthermore, the conductivity of the solution has a direct influence on the ability to electrospin nanofibers as well as the morphology of the fibers. In general, characterization of the electrospinning solution can probe drug-polymer-solvent interactions that may inform the properties of the final solid dispersion and release kinetics.

Several techniques are employed to investigate the bulk properties of the fiber mats or fabrics that emerge from the electrospinning process. The physical properties of a fabric can be accessed by the measurement of density (basis weight), uniformity, surface roughness, and mechanical strength. These properties can influencebothdrug release kinetics andalso user perceptions of a product in clinical trials. Other than these physical properties, specific fiber properties have a directassociation to the release mechanism. For example, fiber size, morphology, arrangement, and surface features individually and alone can impact the drug release kinetics[47]. Scanning electron microscopy (SEM) and transmission electron microscopy (TEM) are standard techniques used to measure fiber size and size distribution, as well as alignment. Microscopy techniques can also be used to visualize crystalline drug dispersed within the polymer fibers. Drug that is localized onto the surface of a fiber often results in a burst release. X-ray photoelectron spectroscopy (XPS) is often used to probe the surface chemistry of a fiber, and is used to quantify the amount of drug enriched at the fiber surface. Other analytical techniques often employed on electrospun nanofibers include differential scanning calorimetry (DSC) and X-ray diffraction (XRD), which canprobe drug-polymer compatibility and crystallinity in the finished fibers. Fourier transform infrared spectroscopy (FTIR) and Raman spectroscopyare also useful tools toexamine the molecular configurations of the drug and polymer in the resulting fibers. In general, these analytical techniques are useful for probing the bulk properties of a fabric to understand their effect on drug release from the final solid dispersions.

\section{Uniaxial fiber design considerations for sustained release}

\subsection{Uniaxial electrospinning process and solution properties}


Uniaxial electrospun fibers represent a relatively simple and scalable method to achieve rapid and sustained release of encapsulated drugs. A bench-topscale uniaxial electrospinning setup requires a micropump, syringe, syringe needle, power generator, metal collector, and polymer solution. Briefly, a chosen polymer solution is added to the needle-capped syringe, which is then attached to a micropump. The micropump is directed towards a grounded metal collecting electrode, at a given distance, at which point the positive lead of the power generator is attached to the syringe needle. As the polymer solution is extruded through the syringe needle a voltage is applied to create an electric field from the micropump to the metal collector. The electric field pulls the extruded polymer solution into a single instable Taylor cone, which is then whipped towards the metal electrode. This process causes rapid evaporation of solvent yielding thin solid polymer fibers collected on the metal collector.

Processing parameters and formulation properties represent important design elements that can impact the development of sustained release fibers. Alteration of polymer concentration, electric field strength, feed rate, and collector distance can potentially alter the final finished fiber solid drug dispersion, which in turn can affect the drug release kinetics. Xie and Buschle-Diller[48] showed the effect of fiber diameter, which can be tuned by electrospinning processing and solution parameters, on diffusional release of $2 \%$ tetracycline from PDLLA fibers. In the study, the group incorporated increasing amounts of methanol as a cosolvent to control the resulting fiber diameters. A 1:16 or 1:4 ratio of cosolvent/solvent produced $\sim 800 \mathrm{~nm}$ and $\sim 200 \mathrm{~nm}$ fibers, respectively. Release studies indicated that larger diameter fibers yielded slower release compared to smaller fibers. After $24 \mathrm{~h}$, smaller diameter fibers released threefold more tetracycline than larger diameter fibers. However, the group also studied the effect of fiber diameter on release of $2 \mathrm{wt} \%$ loaded chlorotetracycline from PDLLA electrospun fibers. Results showed that $\sim 1550 \mathrm{~nm}$ fibers release chlorotetracycline faster than $200 \mathrm{~nm}$ fibers. These differences in drug release as a function of fiber diameter may be a result of swelling behavior and drug solubility in the different fiber formulations. Other studies have also shown differing effects of fiber diameter on release behavior $[49,50]$. Verreck et al. [50] posited that smaller diameter fibers are more tightly packed, inhibiting the rate of matrix swelling. It would be expected that for pure diffusional release, larger diameter fibers would create a longer path length for drug to diffuse, but these studies remind us that drug release mechanisms are often very complex. Formulation properties such as polymer concentration and solvent choice can directly affect electrospin processing and resulting drug dispersion in the fibers [50]. Therefore, formulation properties and processing parameters must be considered simultaneously when developing uniaxial sustained electrospun fibers.

\subsection{Effect of polymer and drug dispersion on sustained release}

In contrast to burst release fibers, sustained release fibers require extensive design considerations depending on the desired drug release application. Because burst release fibers generally incorporate fast dissolving polymers, which quickly hydrate in aqueous solution and dump their drug load [25-27, 51-52], design of these systems are relatively simple when compared to design constraints necessary for sustained release applications.Briefly, major design elements may include polymer choice, drug physiochemical properties, drug-polymer compatibility, formulation 
properties, and electrospinning processing parameters. The interplay of each aforementioned design parameters can significantly affect the release profile of drug loaded-electrospun fibers. The mechanisms of sustained release from electrospun fibers can be complex. However, the rate of matrix hydration and drug diffusion out of polymer has been the primary focus of many studies $[40,53]$. In this section, we discuss how each of these aspects contributes to the design of sustained release uniaxial electrospun fibers.
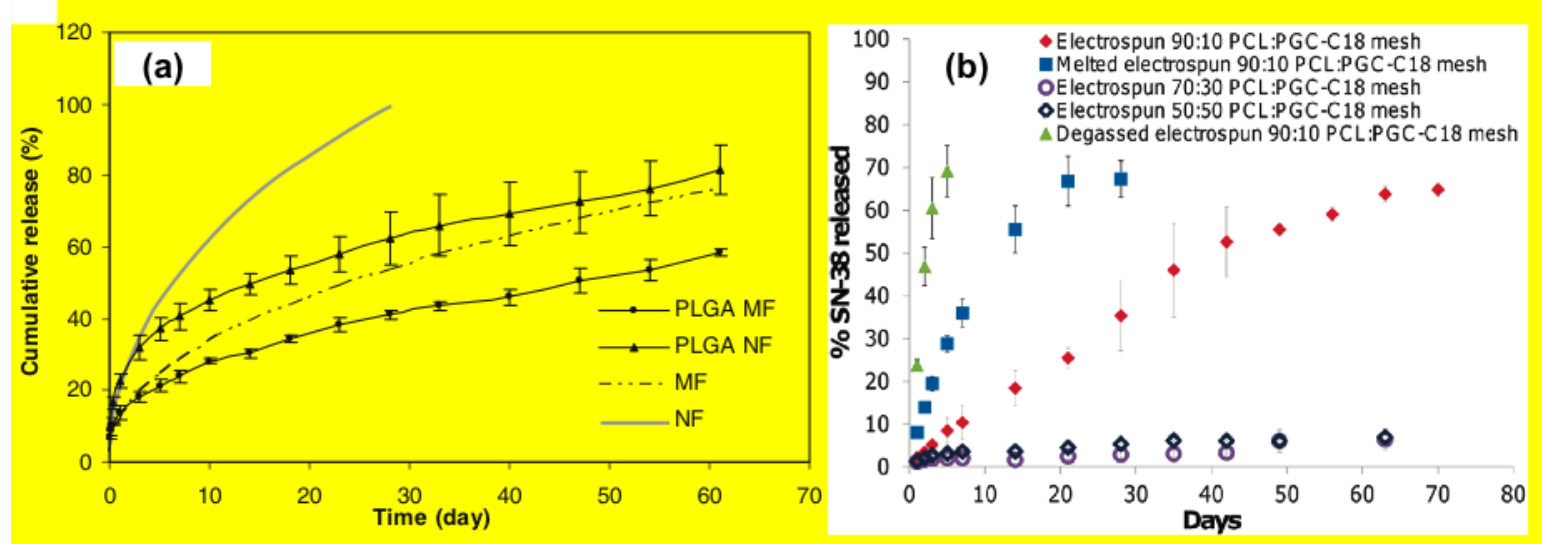

Figure 1. Examples of sustained release curves from electrospun uniaxial fibers: (a) 10 wt\% loaded paclitaxel in PLGA microfibers (PLGA MF) and nanofibers (PLGA NF) and first order release curve fitting for microfibers (MF) $(k=0.119, n=0.45)$ and nanofibers (NF) $(k=0.223, n=0.45)$. Release of paclitaxel sustained for 60 days to $80 \%$ cumulative release in PLGA NF and $60 \%$ cumulative release in PLGA MF. Fiber diameter significantly affected the release profile.[54]; (b) Release curves of SN-38, a bioactive agent, from blends of PCL and poly (glycerol monostearate-co- $\varepsilon$-caprolactone) (PGC-C18) at ratios of 90:10, 70:30, 50:50 PCL:PGC-C18. Effect of air displacement was minimal for the degassed 90:10 PCL:PGC-C18 mesh comparing to the native one. Melted mesh (at $80^{\circ} \mathrm{C}$ for $1 \mathrm{~min}$ ) reduced the pores and further eliminated time required for air displacement comparing to the native one. Higher PGC-C18 doping in the mesh significantly reduce the release rate. The effect on air displace has a direct influence on the wetting of the mesh and thus affects the release rate. [40]

As mentioned previously, PLGA is a well-established electrospun fiber system for sustained release of hydrophobic and hydrophilic compounds. Xie and Wang [54]showed the sustained release of paclitaxel, a lipophilic anti-cancer drug, out to 60 days from PLGA electrospun fibers loaded at $10 \mathrm{wt} \%$. The same PLGA fiber used by Kim et al. [55] demonstrated controlled release of cefoxitin sodium salt, a hydrophilic antibacterial drug incorporated at $5 \mathrm{wt} \%$. However, in this study the results showed that $1 \mathrm{wt} \%$ and $5 \mathrm{wt} \%$ loaded cefoxitin sodium salt resulted in a significant burst portion within the first hour of release from PLGA fibers. Incorporation of PLA/PEG-b-PLA into PLGA yielded continuous sustained release of $27 \%$ of loaded drug over one week, after an initial burst phase. These examples show the use of PLGA for long-term release of a hydrophobic drugout to two months and sustained release of a hydrophilic drug out to one week. However, significant gaps in sustained release remain, particularly related to the sustained release of hydrophilic molecules. The study by Kim et al. [55] still shows significant burst release ( $50-70 \%$ in first hour), and at $5 \mathrm{wt} \%$, the loading is still low 
compared to what would be needed for many clinical applications, which often require high doses and realistic total dosage sizes.

Yohe et al.[40] demonstrated that the use of superhydrophic electrospun fibers could control air-displacement and sustain release of a $1 \mathrm{wt} \%$ small molecule hydrophobic drug past 60 days. This study focused on controlling the rate of matrix hydration as a means of sustaining drug release. In this study, the mechanism of drug release relied on the accessibility of drug in polymer to media. The high glass transition temperatures of polymers such as PLGA provide a different mechanism of sustained release, on a molecular level, by inhibiting drug diffusion through polymer chains. This mechanism hinges on the dispersal of drug molecules through polymer networks within single electrospun fibers. Lyu et al. [56] showed the effect of polymer Tg on drug release. In the study, hydrophobic dexamethasone was loaded at $10 \mathrm{wt} \%$ into two polyurethanes with low and high glass transition temperatures. The results showed that diffusional drug release was much slower for the high $\mathrm{Tg}$ polyurethane and much faster in the low $\mathrm{Tg}$ polyurethane. Furthermore, blends of the two polymers yielded intermediate release of the hydrophobic drug. The examples above incorporating hydrophobic drugs into fibers showed sufficient sustained release but again are limited by the relatively low drug loading used in the fibers.

An important factor associated with sustained release from uniaxial matrix fibers is drug-polymer compatibility [57]. Drug polymer compatibility refers to the physical interaction between drug molecules and polymer chains. The degree to which drug and polymer are compatible will directly affect the drug distribution in the final solid drug dispersion. This compatibility is primarily dependent on drug solubility in the polymersolvent system. Zeng et al. [57] demonstrated the effect of drug compatibility on release by loading $15 \mathrm{wt} \%$ lipophilic paclitaxel and $1.6 \mathrm{wt} \%$ hydrophilic doxorubicin hydrochloride into PLLA electrospinning solution. Paclitaxel was highly soluble in the organic solvent used in the formulations whereas doxorubicin hydrochloride showed low solubility. As a result, during electrospinning the rapid evaporation of solvent allowed paclitaxel to be fully dispersed and encapsulated within the finished solid fibers. Because doxorubicin hydrochloride was not soluble, phase separation occurred during the spinning process that resulted in a large portion of the drug on the surface of the fibers, causing higher burst release. Conversion of doxorubicin hydrochloride to a lipophilic drug by addition of ammonia resulted in better solubility in solution and improved encapsulation in the finished fiber. Seif et al.[58] further investigated drugpolymer compatibility and how it affects drug crystallization and subsequent release. The study showed that the tendency of $\sim 10$ wt\% hydrophilic caffeine, loaded in PCL, to crystallize was dependent on both the polymer-drug compatibility as well the solvent selection. Furthermore, the results of the study demonstrated that release of caffeine from PCL was dampened in fibers without surface crystallized drug compared to fibers with clear drug crystals.

The ionization state of incorporated drugs can also influence drug distribution within the finished fibers. The study presented by Kim et al. above suggested that a major portion of incorporated cefoxitin sodium salt localized to the surface because of the high ionic strength of the drug [55]. Ball and Woodrow[32] demonstrated the PVP fiber surface enrichment of highly loaded lipophilic maraviroc (28 wt\%), through XPS analysis. In this study it was suggest that physiochemical properties of the drug as well 
as drug loading can influence the propensity of drug to localize to the surface of fibers. A formulation with low drug-polymer compatibility resulting from drug solubility, ionization state, and loading will result in drug partitioning to the surface of the fibers which can then lead to burst release. Drug loading also plays a key role in the propensity of drug to localize to the surface of fibers. Several studies have shown the influence of higher drug loadings on increased burst release [59-61]. Therefore, much of the small molecule sustained release demonstrated has been accomplished by using low loadings and hydrophobic molecules to improve compatibility with the required solution properties of hydrophobic sustained release polymers [53,62]. Recent advances have led to the development of more complex platforms to sustain release of compounds $[63,64]$. However, sustained release from uniaxial electrospun fibers to sustain release is strongly influence by drug-polymer-solvent compatibility as it relates to the hydration of the fiber matrix and drug dissolution.

As discussed previously, sustained release from electrospun uniaxial fibers can be significantly manipulated through a number of platform and formulation characteristics. Polymer choice is the first step in developing a sustained release system where its hydrophobicity and $\mathrm{Tg}$ value substantially influence drug release kinetics. Drug compatibility with the polymer-solvent system is also an important design parameter for sustained release from uniaxial fibers. Low drug solubility in the polymersolvent system can cause preferential localization of drug to the surface of fibers during electrospinning even at low loading, which leads to burst release. Drug loading and ionization state can also influence encapsulation of drug in fibers. Higher drug loading and ionic content have been shown to lead to surface localization of drug. Processing parameters can also influence the morphology of finished fibers, which can affect resulting release. The complexity of these design parameters limits the ability to achieve sustained release with a wide variety of drugs, especially at high loadings. Therefore, much of the sustained release observed from uniaxial fibers has been achieved with low loadings and hydrophobic molecules. An optimized combination of the above mentioned design parameters could provide ideal sustained release for both hydrophilic and hydrophobic small molecule drugs at high loadings.

\section{Coaxial fiber design considerations for sustained release}

\subsection{Coaxial electrospinning process and solution properties}

Core-shell fibers produced by coaxial electrospinning are an alternative strategy that has been explored to achieve sustaining drug release from electrospun materials. Coaxial electrospinning is similar to the process for forming uniaxial fibers except for the nozzle configuration. A coaxial nozzle comprisestwo concentric but separate nozzles, which are individually controlled by a separate syringe pump. Thissetup allowsfor different solutions to be used in each nozzle as well as separate flow rate control. The core-shell fiber architecture is a result of the compound Taylor cone formed by this coaxial nozzle geometry.For sustained release applications, drugs are typically loaded into the polymer solution that forms the inner core whereas the outer shellconsists of a polymer that serves as a diffusive barrier for the drugs. Since coaxial electrospinning involves the use of two polymer solutions, the miscibility of the polymers and the solvents used in the core and the shell solution becomes a very important factor that 
impacts the integrity of the final core-shell architecture. Another important variable is the solution flow rate of the shell and core polymers, which can becontrolled to set the shell thickness and core diameter.In general, electrospun coaxial fibers often have the advantages in sustained drug release bythe localization of drug in the inner core and by the ability to tune the shell thickness.

\subsection{Effect of core-shell composition and architecture on sustained release}

As mentioned previously (section 4.1), coaxial fibers have the ability to tune the release profile over a wide range of time scales due to the core-shell structure.Although a number of reports have described the fabrication and application of various core-shell fibers for drug release (Table 1), the process can require significant optimization torealize sustained release from different core, shell and drug compositions. Here, we consider factors that are most important to the structure of the coaxial fibers and their sustained drug release.

\subsubsection{Integrity of the core-shell structure}

The release behavior of drugs from core-shell fibers is highly dependent on the distribution of the drug within the core or shell phase, as well as the final morphology of the compound fibers. Solvent volatility and evaporation rate are of particular importance to produce uniform fiber diameter and regular morphology of core-shell fibers. Katsogiannis et al. [65] describe solvent mixtures of chloroform, dichloromethane, tetrahydrofuran, and formic acid with DMSO to produce PCL fibers with a porous structure. Volatile solvents have faster evaporation rates than DMSO. After the remaining DMSO evaporates, a porous fiber structure is obtained. Since coaxial fibers usually consist of two different types of polymer solutions and solvents, the mixture of solvent at the Taylor cone is unavoidable. Dayal and Kyu [66] have demonstrated that when core and shell solutions are immiscible, andthe shell solvent evaporates faster than the core solvent, a hollow fiber is obtained. Even if a hollow fiber structure is avoided, insufficient interfacial compatibility of the core and shell solutions often results in delamination at the interface. In contrast, if the two solvents are miscible and the evaporation rate of the shell solvent is faster than the core solvent, the entrapped core solvent may dissolve some portion of the shell polymer and produce a porous shell structure. Therefore, choosing the compatible solvents and polymer solutions is a crucial step to form coaxial fibers with great integrity.

The shell layer of coaxial fibers can often contain some amount of drugs from the core as a result of mixing of the core-shell solvents during electrospinning or due to defects in the core-shell structure. These flaws in the integrity of the core-shell architecture can compromise sustained release and result in burst release of the drug entrapped within the core.For example, Sohrabiet al. [67]showed that the release of the ampicillinloaded in the core of the poly(methyl methacrylate)-nylon6 (core-shell) fibers exhibited an initial burst release of $30 \%$ of the cumulative release. They suggested that the initial burst effect is most likely due to the accumulation of the drug molecules at or near the surface of the fibers during coaxial electrospinning. In addition, higher concentrations of the ampicillin in the core magnify the accumulation of the drug molecules at or near the surface of the fibers resulting in the initial burst. In a separate example, Tiwari et al. [68] showed the release profiles of metoclopramide hydrochloride 
from PVA-PCL (core-shell) fibers exhibited an initial burst to about $55 \%$ of the total release. They suggested that the burst effect is most likely due to the presence of pores (either micron or nano-sized) in the PCL shell, which is similar to the release mechanism found by others $[69,70]$. Therefore, in order to achieve sustained release from core-shell fibers, the controlling of the shell structure is an important step.

\subsubsection{Shell thickness and composition}

Once the structural integrity of the core-shell architecture is achieved to localize the drug in a corethat is surrounded by a uniform and smooth shell, control of shell thickness and composition can be further explored to modulate release. In core-shell fibers, most reports suggest that the sustained releaseprofiles are usually a contribution from the rate-limiting effect of drug diffusion through the shell polymer [25,70-74]. Therefore, the thickness and composition of the shell in coaxial fibers play important roles since both affect how well the drug is encapsulated in the core and the wetting behavior of the coaxial fibers during release. Wang et al. [71] used hydrophobic PLA and poly(3-hydroxy butyrate) (PHB) to produce coaxial fibers loaded with dimethyloxalylglycine (DMOG). They observed that coaxial fibers made of PHB-core and PLA-shell exhibited a burst release whereas those made of PLA-core and PHBshell exhibited a two-phase sustained release over 30 days. The first phase of the release was shown to be independent of the shell thickness. However, the second phase showed a linear release that was sustained over 30 days andwas dependent on the shell thickness. For example, thin shells $(\sim 120 \mathrm{~nm})$ showed $\sim 70 \%$ cumulative drug release in 11 days, whereas thick shell $(\sim 230 \mathrm{~nm})$ required $>30$ days to reach the same cumulative percent release. In this example, sustained release of hydrophilic small molecules from coaxial fibers strongly depends on the hydrophobicity of the shell layer and the thickness of the shell.

Llorens et al.[75] compared the release of triclosan (an antibacterial drug) and curcumin (an anti-carcinogenic drug) loaded in the core of coaxial fibers made from PEG and poly(butylene succinate). Their results showed thatdrug release was higher from poly(butylene succinate)-core and PEG-shell fibers than core-shell fibers of the opposite composition. In addition, they observed that drug releases from core-shell fibers were associated with a controlled wettingmechanism. This was supported by a significant increase in the amount of triclosan and curcumin release upon use of low surface tension release media such as mixtures containing ethanol [75].In another study reported by He et al. [76], metronidazole was loaded up to $35 \mathrm{wt} \%$ in PCL-core fibers surrounded by a gelatin-shell.Contact angle measurementsshowed that the gelatin-shell could be made more hydrophobicby crosslinking, and this resulted in sustained release of metronidazole for up to 6 days.In contrast, an uncross-linked gelatin-shell resulted in $80 \%$ of cumulative release after 1day.Overall, there is a significant dependence on the surface hydrophilic-hydrophobic properties of the coaxial fibers and the sustained release of drugs. 
(a)

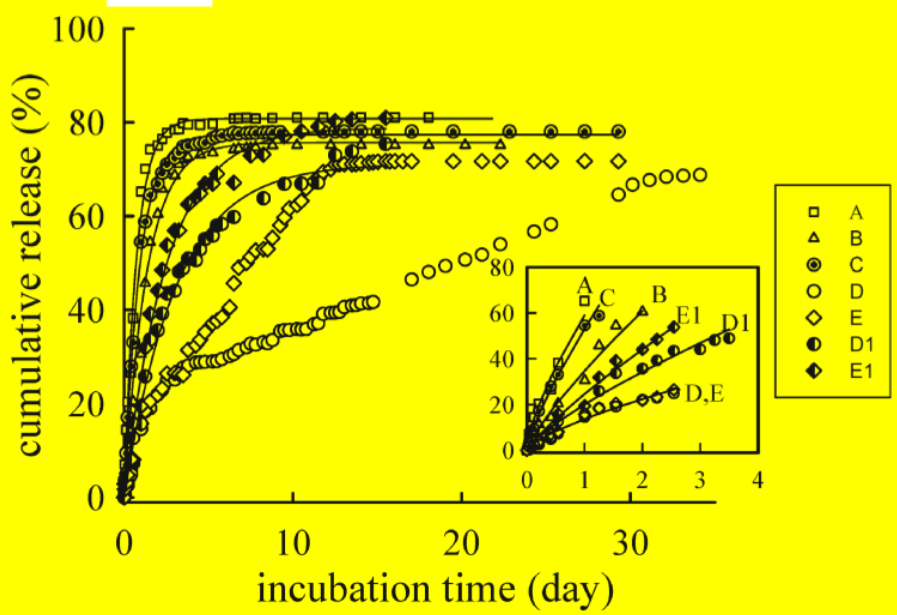

(b)

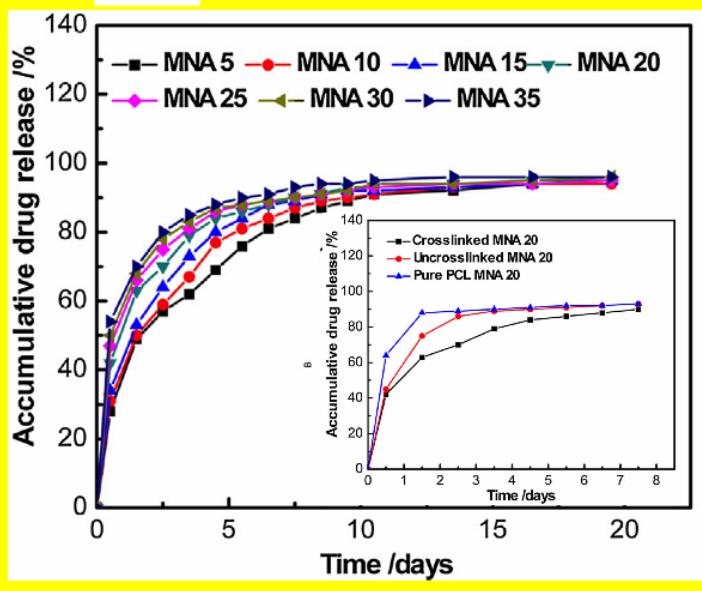

Figure 2. Examples of sustained release curves from electrospun coaxial fibers: (a) Cumulative release of DMOG from electrospun fibers. Figure legends: $A(P L A), B$ (PHB), C (PLA-core PHB-shell; Qc/Qs = 0.5/1.5; drug in shell), D (PLA-core PHB-shell; $\mathrm{Qc} / \mathrm{Qs}=0.5 / 1.5$; drug in core), $\mathrm{E}$ (PLA-core PHB-shell; Qc/Qs = 2.0/1.5; drug in core), D1 (PHB-core PLA-shell; Qc/Qs = 1.5/0.5; drug in core), E1 (PHB-core PLA-shell; $\mathrm{Qc} / \mathrm{Qs}=1.5 / 2.0$; drug in core). Fiber type $\mathrm{D}$ showed a sustained release of $70 \%$ of DMOG over 30 days. Switching core and shell polymers resulted in burst release due to different hydrophobicity of PLA and PHB. Shell thickness of fiber type D is twice thicker than fiber type $E$ resulting in significant effect in release rate. [71]; (b) Cumulative release of metronidazole (MNA) loaded from $5 \mathrm{wt} \%$ to $35 \mathrm{wt} \%$ in the core of PCL-core gelatin-shell fibers (all gelatin shells were cross-linked). Higher drug loading increased the release rate. Insert showed the release curves on the effect gelatin shell crosslinking. Crosslinking of the gelatin shell resulted in changes of hydrophobicity and therefore changing the release behavior. [76]

\section{Conclusions and future perspectives}

We have highlighted the design considerations to achieve sustained release from electrospun uniaxial and coaxial fibers. In particular, we focused our discussion on current strategies that may be promising for weekly or monthly release of hydrophilic drugs at multimilligram quantities per day. In addition, several design constrains need to be considered for fibers that contain high loading of drugs. For example, sustained release from uniaxial fibers can be greatly affected by polymer choice (where the most important factors are hydrophobicity and $\mathrm{Tg}$ of the polymer), polymer and drug compatibility, drug loading and ionization state, and processing parameters. Specifically, Carson et al. [42] demonstrated blended uniaxial fibers to control release of a 10-40\% hydrophilic small molecule past 10 days. This study demonstrated a multifaceted release mechanism that used the hydrophobic nature of the polymers and different polymer's Tg to control release of a hydrophilic drug. In general, it appears that it is very difficult to reach the specified targets (20 wt\% loading, 10-100 milligram daily release for greater than a week) listed for prolonged release of small molecule hydrophilic drugs from uniaxial fibers. Uniaxial electrospun fiber strategies in the future will likely need to focus on blended compositions to realize the complex design constraints associated 
with sustained release of small water-soluble molecules. In contrast, sustained release from coaxial fibers is greatly influenced by the integrity of the coaxial fiber structure and the shell composition and thickness. Based on the mechanisms of release associated with the two fiber types described above, coaxial fibers are more likely to achieve the required targets for sustained release of small hydrophilic drugs. The ability to load high amounts of drug in a core with a tunable shell is a major advantage when compared to sustained release efforts from uniaxial fibers. In general, electrospinning shows promise for developing sustained release materials with tunable release kinetics that depend upon material surface chemistry, drug loading, and processing parameters. Our mechanistic understanding of hydrophilic drug release from uniaxial and coaxial fibers offers insights to develop more robust electrospun drug release systems.

\section{Acknowledgement}

This work is supported by a grant from the US National Institutes of Health (AI094412)and a grant from the Bill and Melinda Gates Foundation (1067729), which were awarded to K.A.W.

\section{References}

[1] A. Furmhals, US patent 1,975,504, 1934.

[2] R. Gopal, S. Kaur, Z. Ma, C. Chan, S. Ramakrishna, T. Matsuura, Electrospun nanofibrous filtration membrane, J. Membr. Sci. 281 (2006) 581-586.

[3] D. Aussawasathien, C. Teerawattananon, A. Vongachariya, Separation of micron to sub-micron particles from water: electrospun nylon-6 nanofibrous membranes as prefilters, J. Membr. Sci. 315 (2008) 11-19.

[4] H. Schreuder-Gibson, P. Gibson, K. Senecal, M. Sennett, J. Walker, W. Yeomans,

D. Ziegler, P.P. Tsai, Protective textile materials based on electrospun nanofibers, J. Adv. Mater. 34 (2002) 44-55.

[5] Z. Gu, Q. Xu, Cosmetic mask based on electrospinning nanometer fiber for skin, comprises electrospinning nanometer fiber nonwoven fabric or nonwoven felt, CN101390814-A.

[6] N. Kusamoto, T. Tajima, Fiber comprises an eggshell membrane component useful for producing a fiber assembly, which is used as a wound dressing or a cosmetic sheet. US patent, US2009031691-A1.

[7] S.E. Chung, C.H. Park, Manufacture of shape memory polyurethane electrospun web for e.g. protective clothing, involves electrospinning solution obtained by adding poly(caprolactone diol), diphenylmethane diisocyanate and butanediol to polyurethane, US patent KR2010048661-A.

[8] S. Lee, S.K. Obendorf, Developing protective textile materials as barriers to liquid penetration using melt-electrospinning, J. Appl. Polym. Sci. 102 (2006) 3430-3437. [9] M. Gorji, A.A.A. Jeddi, A.A. Gharehaghaji, Fabrication and characterization of polyurethane electrospun nanofiber membranes for protective clothing applications, J. Appl. Polym. Sci. 125 (2012) 4135-4141.

[10] R. Rojas, N.J. Pinto, Using electrospinning for the fabrication of rapid response gas sensors based on conducting polymer nanowires, IEEE Sens. J. 8 (2008) 951-953.

[11] T. Kowalczyk, A. Nowicka, D. Elbaum, T.A. Kowalewski, Electrospinning of bovine serum albumin. Optimization and the use for production of biosensors, Biomacromol. 9 
(2008) 2087-2090.

[12] Z. Dong, S.J. Kennedy, Y. Wu, Electrospinning materials for energy-related applications and devices, J. Power Sources 196 (2011) 4886-4904.

[13] Q.P. Pham, U. Sharma, A.G. Mikos, Electrospinning of polymeric nanofibers for tissue engineering applications: a review, Tissue Eng. 12 (2006) 1197-1211.

[14] X. Hu, S. Liu, G. Zhou, Y. Huang, Z. Xie, X. Jing,Electrospinning of polymeric nanofibers for drug delivery applications,J. Control. Release 185 (2014) 12-21.

[15] T. Subbiah, G.S. Bhat, R.W. Tock, S. Parameswaran, S.S.

Ramkumar,Electrospinning of nanofibers,J. Appl. Polym. Sci. 96 (2005) 557-569.

[16] F.L. Zhou, R.H. Gong, I. Porat, Mass production of nanofibre assemblies by electrostatic spinning, Polym. Int. 58 (2009) 331-342.

[17] L. Persano, A. Camposeo, C. Tekmen, D. Pisignano, Industrial upscaling of electrospinning and applications of polymer nanofibers: a review, Macromol. Mater. Eng. 298 (2013) 504-520.

[18] C.J. Luo, S.D. Stoyanov, E. Stride, E. Pelan, M. Edirisinghe, Electrospinning versus fibre production methods: from specifics to technological convergence, Chem. Soc.Rev. 41 (2012) 4708-4735.

[19] P. Zahedi, I. Rezaeian, S.O. Ranaei-Siadat, S.H. Jafari, P. Supaphol, A review on wound dressings with an emphasis on electrospun nanofibrous polymeric bandages, Polym. Adv. Technol. 21 (2010) 77-95.

[20] M.S. Khil, D.I. Cha, H.Y. Kim, I.S. Kim, N. Bhattarai, Electrospun nanofibrous polyure- thane membrane as wound dressing, J. Biomed. Mater. Res. B. 67B (2003) 675-679.

[21] K.A. Rieger, N.P. Birch, J.D. Schiffman, Designing electrospun nanofiber mats to promote wound healing - a review, J. Mater. Chem. B. 1 (2013) 4531-4541.

[22] V. Pillay, C. Dott, Y.E. Choonara, C. Tyagi, L. Tomar, P. Kumar, L.C. du Toit, V.M.K. Ndesendo, A review of the effect of processing variables on the fabrication of electrospun nanofibers for drug delivery applications, J. Nanomater. (2013) 1-22. [23] T.J. Sill, H.A. von Recum, Electrospinning: applications in drug delivery and tissue engineering, Biomater. 29 (2008) 1989-2006.

[24] H.S. Yoo, T.G. Kim, T.G. Park, Surface-functionalized electrospun nanofibers for tissue engineering and drug delivery, Adv. Drug Delivery Rev. 61 (2009) 1033-1042. [25]C. Ball, K.A. Woodrow, Electrospun solid dispersions of maraviroc for rapid intravaginal preexposure prophylaxis of HIV, Antimicrob. Agents Chemother.58 (2014) 4855-4865.

[26] A.K. Blakney, E.A. Krogstad, Y.H. Jiang, K.A. Woodrow, Delivery of multipurpose prevention drug combinations from electrospun nanofibers using composite microarchitectures, Int. J. Nanomed.9 (2014) 2967-2978.

[27] E.A. Krogstad, K.A. Woodrow, Manufacturing scale-up of electrospun poly(vinyl alcohol) fibers containing tenofovir for vaginal drug delivery, Int. J. Pharm.475 (2014) 282-291.

[28] C. Ball, E. Krogstad, T. Chaowanachan, K.A. Woodrow, Drug-eluting fibers for HIV1 inhibition and contraception, PLoS ONE 7 (2012) e49792.

[29] A.K. Blakney, C. Ball, E.A. Krogstad, K.A. Woodrow, Electrospun fibers for vaginal anti-HIV drug delivery, Antiviral. Res. 100 (2013) S9-16. 
[30] S.C. Sundararaj, M.V. Thomas, R. Peyyala, T.D. Dziubla, D.A. Puleo, Design of a multiple drug delivery system directed at periodontitis, Biomater. 34 (2013) 8835-8842. [31] E.J. Falde, J.D. Freedman, V.L.M. Herrera, S.T. Yohe, Y.L. Colson, M.W. Grinstaff, Layered superhydrophobic meshes for controlled drug release, J. Control. Release 214 (2015) 23-29.

[32] C. Ball, K.A. Woodrow, Electrospun fibers for microbicide drug delivery, in: J. das Neves, B. Sarmento (Eds.), Delivery Science and Technology, Pan Stanford Publishing Pte. Ltd., 2014, 459-499.

[33] R.C. Owens, A.F. Shorr, Rational dosing of antimicrobial agents: pharmacokinetic and pharmacodynamic strategies, Am. J. Health-Syst. Pharm. 66 (2009) S23-S30. [34] W.R. Spreen, D.A. Margolis, J.C. Pottage Jr., Long-acting injectable antiretrovirals for HIV treatment and prevention, Curr. Opin. HIV AIDS 8(2013), 565-571.

[35]J. Xie, C.H. Wang, Electrospun micro- and nanofibers for sustained delivery of paclitaxel to treat C6 glioma in vitro, Pharm. Res. 23 (2006) 1817-1825.

[36] G. Verreck, I. Chun, J. Rosenblatt, J. Peeters, A.V. Dijck, J. Mensch, M. Noppe, M.E. Brewster, Incorporation of drugs in an amorphous state into electrospun nanofibers composed of a water-insoluble, nonbiodegradable polymer, J. Control. Release 92 (2003) 349-360.

[37] P.F. McDonald, J.G. Lyons, L.M. Geever, C.L. Higginbotham, In vitro degradation and drug release from polymer blends based on poly(DL-lactide), poly(L-lactideglycolide) and poly(e-caprolactone), J. Mater. Sci. 45 (2010) 1284-1292.

[38] E. Piskin, N. Bolgen, S. Egri, I.P. Isoglu,Electrospun matrices made of poly(alphahydroxy acids) for medical use,Nanomed. 2 (2007)441-57.

[39] J.J. Zhang, J. Liu, H. Yu, Y. Zhang, M.F. Zhu, Y.M. Chen, Crosslinked electrospun UPM/PHBV/PVP fibers for sustained drug release, Mater. Sci. Forum 610-613 (2009), 1331-1334.

[40] S.T. Yohe, Y.L. Colson, M.W. Grinstaff, Superhydrophobic materials for tunable drug release: using displacement of air to control delivery rates, J. Am. Chem. Soc. 134 (2012) 2016-2019.

[41] M. Zamani, M.P. Prabhakaran, S. Ramakrishna, Advances in drug delivery via electrospun and electrosprayed nanomaterials, Int. J. Nanomed. 8 (2013) 2997-3017. [42] D. Carson, Y. Jiang, K.A. Woodrow, Tunable release of multiclass anti-HIV drugs that are water soluble and loaded at high drug content in polyester blended electrospun fibers, Pharm Res. (2015) 1-12.

[43] C. Huang, S. Soenen, E. van Gulck, G. Vanham, J. Rejman, S. Van Calenbergh, et al., Electrospun cellulose acetate phthalate fibers for semen induced anti-HIV vaginal drug delivery, Biomater. 33 (2012) 962-969.

[44] W.W. Graessley, The entanglement concept in polymer rheology, Adv. Polym. Sci.16 (1974) 1-179.

[45] A.C. Stijnman, I. Bodnar, R.H. Tromp, Electrospinning of food-grade polysaccharides, Food Hydrocolloids25 (2011) 1393-1398.

[46] A. Szentivanyi, U. Assmann, R. Schuster, B. Glasmacher, Production of biohybrid protein/PEO scaffolds by electrospinning, Materialwiss. Werkst.40 (2009) 65-72.

[47] Z.X. Meng, W. Zheng, L. Li, Y.F. Zheng, Fabrication, characterization and in vitro drug release behavior of electrospun PLGA/chitosan nanofibrous scaffold, Mater. 
Chem. Phys. 125 (2011) 606-611.

[48] Z. Xie, G. Buschle-Diller, Electrospun poly(D,L-lactide) fibers for drug delivery: the influence of cosolvent and the mechanism of drug release, J. Appl. Polym. Sci. 115 (2010) 1-8.

[49] S.C. Chen, X.B. Huang, X.M. Cai, J. Lu, J. Yuan, J. Shen, The influence of fiber diameter of electrospun poly(lactic acid) on drug delivery, Fibers Polym. 9 (2012) 11201125.

[50] G. Verreck, I. Chun, J. Peeters, J. Rosenblatt, M.E. Brewster, Preparation and characterization of nanofibers containing amorphous drug dispersions generated by electrostatic spinning, Pharm. Res. 20 (2003) 810-817.

[51] D.G. Yu, X.X. Shen, C. Branford-White, K. White, L.M. Zhu, S.W.A. Bligh, Oral fastdissolving drug delivery membranes prepared from electrospun polyvinylpyrrolidone ultrafine fibers, Nanotechnol. 20 (2009) 055104.

[52] X. Li, M.A. Kanjwal, L. Lin, I.S. Chronakis, Electrospun polyvinyl-alcohol nanofibers as oral fast-dissolving delivery system of caffeine and riboflavin, Colloids Surf. B 103 (2013) 182-188.

[53] S. Fredenber, M. Wahlgren, M. Reslow, A. Axelsson, The mechanisms of drug release in poly(lactic-co-glycolic acid)-based drug delivery systems-A review, Int. J. Pharm. 415 (2011) 34-52.

[54]J. Xie, C.H. Wang, Electrospun micro- and nanofibers for sustained delivery of paclitaxel to treat C6 glioma in vitro, Pharm. Res. 23 (2006) 1817-1825.

[55] K. Kim, Y.K. Luu, C. Chang, D. Fang, B.S. Hsiao, B. Chu, M. Hadjiargyrou, Incorporation and controlled release of a hydrophilic antibiotic using poly(lactide-coglycolide)-based electrospun nanofibrous scaffolds, J. Control. Release 98 (2004) 4756.

[56] S. Lyu, R. Sparaer, C. Hobot, K. Dang, Adjusting drug diffusivity use miscible polymer blends, J Control. Release 102 (2005) 679-687.

[57] J. Zeng, L.X. Yang, Q.Z. Liang, X.F. Zhang, H.L. Guan, X.L. Xu, X.S. Chen, X.B. Jing, Influence of the drug compatibility with polymer solution on the release kinetics of electrospun fiber formulation, J. Control. Release 105 (2005) 43-51.

[58] S. Seif, L. Franzen, M. Windbergs, Overcoming drug crystallization in electrospun fibers - Elucidating key parameters and developing strategies for drug delivery, Int. J. Pharm. 478 (2015) 390-397.

[59] M.V. Natu, H.C. de Sousa, M.H. Gil, Effects of drug solubility, state, and loading on controlled release in bicomponent electrospun fibers, Int. J. Pharm. 397 (2010) 50-58.

[60] M. Zamani, M. Morshed, J. Varshosaz, M. Jannesari, Controlled release of metronidazole benzoate from poly e-caprolactone electrospun nanofibers for periodontal diseases, Eur. J. Pharm. Biopharm. 2 (2010) 179-185.

[61] W. Cui, X. Li, X. Zhu, G. Yu, S. Zhou, J. Weng, Investigation of drug release and matrix degradation of electrospun poly(dl-lactide) fibers with paracetanol inoculation, Biomacromol. 7 (2006) 1623-1629.

[62] C. Xie, X. Li, X. Luo, Y. Yang, W. Cui,J. Zou, S. Zhou, Release modulation and cytotoxicity of hydroxycamptothecin-loaded electrospun fibers with 2- hydroxypropylbeta-cyclodextrin inoculations, Int. J. Pharm. 391 (2010) 55-64.

[63] Y.Z. Zhang, X. Wang, Y. Feng, J. Li. C.T. Lim, S. Ramakrishna, Coaxial electrospinning of (fluorescein isothiocyanate-conjugated bovine serum albumin)- 
encapsulated poly(e-caprolactone) nanofibers for sustained release, Biomacromol. 7 (2006) 1049-1057.

[64] C.L. He, Z.M. Huang, X.J. Han, L. Liu, H.S. Zhang, L.S. Chen, Coaxial electrospun poly(I-lactic acid) ultrafine fibers for sustained drug delivery, J. Macromol. Sci. Part B Phys. 45 (2006) 515-524.

[65] K.A.G. Katsogiannis, G.T. Vladisavljević, S. Georgiadou, Porous electrospun polycaprolactone (PCL) fibres by phase separation, Eur. Polym. J. 69 (2015) 284-295.

[66] P. Dayal, T. Kyu, Porous fiber formation in polymer-solvent system undergoing solvent evaporation, J. Appl. Phys. 100 (2006) 043512.

[67] A. Sohrabi, P.M. Shaibani, H. Etayash, K. Kaur, T. Thundat, Sustained drug release and antibacterial activity of ampicillin incorporated poly(methyl methacrylate), Polym. 54 (2013) 2699-2705.

[68] S.K. Tiwari, R. Tzezana, E. Zussman, S.S. Venkatraman, Optimizing partitioncontrolled drug release from electrospun core-shell fibers, Pharm. Nanotechnol. 392 (2010) 209-217.

[69] R. Srikar, A.L. Yarin, C.M. Megaridis, A.V. Bazilevsky, E. Kelley, Desorption-limited mechanism of release from polymer nanofibers, Langmuir 24 (2008) 965-974.

[70] T.T.T. Nguyen, C. Ghosh, S.G. Hwang, N. Chanunpanich, J.S. Park, Porous core/sheath composite nanofibers fabricated by coaxial electrospinning as a potential mat for drug release system, Inter. J. Pharm. 439 (2012) 296-306.

[71] C. Wang, K.W. Yan, Y.D. Lin, P.C.H. Hsieh, Biodegradable core/shell fibers by coaxial electrospinning: processing, fiber characterization, and its application in sustained drug release, Marcomol. 43 (2010) 6389-6397.

[72] L. Viry, S.E. Moulton, T. Romeo, C. Suhr, D. Mawad, M. Cook, G.G. Wallace, Emulsion-coaxial electrospinning: designing novel architectures for sustained release of highly soluble low molecular weight drugs, J. Mater. Chem. 22 (2012) 11347-11353.

[73] T. Kiatyongchai, S. Wongsasulak, T. Yoovidhya, Coaxial electrospinning and release characteristics of cellulose acetate-gelatin blend encapsulating a model drug, J. Appl. Polym. Sci. 131 (2014).

[74] R.K. Malcolm, C.J. Forbes, L. Geer, R.S. Veazey, L. Goldman, P.J. Klasse, J.P. Moore, et al., Pharmacokinetics and efficacy of a vaginally administered maraviroc gel in rhesus macaques, J. Antimicrob. Chemother. 68 (2013) 678-683.

[75] E. Llorens, H. Ibañez, L.J. del Valle, J. Puiggalí, Biocompatibility and drug release behavior of scaffolds prepared by coaxial electrospinning of poly(butylene succinate) and polyethylene glycol, Mater. Sci. Eng. C 49 (2015) 472-484.

[76] M. He, J. Xue, H. Geng, H. Gu, D. Chen, R. Shi, L. Zhang, Fibrous guided tissue regeneration membrane loaded with anti-inflammatory agent prepared by coaxial electrospinning for the purpose of controlled release, Appl. Surf. Sci. 335 (2015) 121129.

[77] E.R. Kenawy, G.L. Bowlin, K. Mansfield, J. Layman, D.G. Simpson, E.H. Sanders, G.E. Wnek, Release of tetracycline hydrochloride from electrospun poly(ethylene-covinylacetate), poly(lactic acid), and a blend, J. Control. Release 81 (2002) 57-64. [78] M. Reise, R. Wyrwa, U. Muller M. Zylinski, A. Volpel, M. Schnabelrauch, A. Berg, K.D. Jandt, D.C. Watts, B.W. Sigusch, Release of metronidazole from electrospun poly (L-lactide-co-D/L-lactide) fibers for local periodontitis treatment, Dent. Mater. 28 (2011) 179-188. 
[79] J.M. Yang, L.S. Zha, D.G. Yu, J. Liu, Coaxial electrospinning with acetic acid for preparing ferulic acid/zein composite fibers with improved drug release profiles, Colloids Surf. B 102 (2013) 737-743.

[80] H. Yu, Y. Jia, C. Yao, Y. Lu, PCL/PEG core/sheath fibers with controlled drug release rate fabricated on the basis of a novel combined technique, Inter. J. Pharm. 469 (2014) 17-22. 
Table 1. Strategies for sustained drug release from electrospun fibers

\begin{tabular}{|c|c|c|c|c|c|c|c|c|c|}
\hline \multirow[b]{2}{*}{ Fiber architecture } & \multicolumn{4}{|c|}{ Agent } & \multicolumn{4}{|c|}{ Release (units) } & \multirow[b]{2}{*}{ Ref. } \\
\hline & Name & $\begin{array}{l}\text { Loading } \\
\text { wt } \%\end{array}$ & $\begin{array}{l}\text { Aq. Sol. } \\
\text { (mg/mL) }\end{array}$ & $\log \mathbf{P}^{\dagger}$ & 1h & $24 h$ & $7 d$ & 14d & \\
\hline \multicolumn{10}{|l|}{ Uniaxial Fibers } \\
\hline \multirow[t]{2}{*}{ PLGA } & Paclitaxel & 10 & $<0.01$ & 3.95 & $10 \%$ & $22 \%$ & $40 \%$ & $50 \%$ & [54] \\
\hline & Cefoxitin sodium & 5 & 0.15 & -0.92 & $70 \%$ & $72 \%$ & $80 \%$ & - & [37] \\
\hline \multirow[t]{3}{*}{ PLA } & $\begin{array}{l}\text { Tetracycline } \\
\text { hydrochloride }\end{array}$ & 5 & $0.46^{\ddagger}$ & $-0.42^{\ddagger}$ & $35 \%$ & $35 \%$ & $35 \%$ & - & [77] \\
\hline & Metronidazole & 40 & 29 & -0.14 & $5 \%$ & $25 \%$ & $45 \%$ & - & [64] \\
\hline & Amoxicillin & 7 & 0.58 & 0.88 & $10 \%$ & $15 \%$ & $20 \%$ & $20 \%$ & [78] \\
\hline \multirow[t]{3}{*}{ PLLA } & Paclitaxel & 15 & $<0.01$ & 3.95 & $0-1 \%$ & $0-1 \%$ & - & - & [57] \\
\hline & $\begin{array}{l}\text { Doxorubicin } \\
\text { hydrochloride }\end{array}$ & 1.6 & $1.18^{\ddagger}$ & $1.41^{\ddagger}$ & $70 \%$ & $87 \%$ & - & - & [57] \\
\hline & Doxorubicin & 1.6 & 0.41 & 0.24 & $20 \%$ & $20 \%$ & - & - & [57] \\
\hline \multirow[t]{2}{*}{ Polyurethane } & Itraconazole & 40 & $<0.01$ & 4.99 & $2 \mathrm{ug} / \mathrm{cm}^{2}$ & $20 \mathrm{ug} / \mathrm{cm}^{2}$ & - & - & [36] \\
\hline & Ketanserin & 10 & $<0.01$ & 3.56 & $2 \mathrm{ug} / \mathrm{cm}^{2}$ & $10 \mathrm{ug} / \mathrm{cm}^{2}$ & - & - & [36] \\
\hline \multicolumn{10}{|l|}{$\begin{array}{l}\text { Coaxial Fibers } \\
\text { (core:shell Qc/Qs) }\end{array}$} \\
\hline \multirow[t]{2}{*}{ PEG:PBS 3/1 } & Triclosan & 5 & $<0.01$ & 5.34 & $75 \%$ & - & - & - & [75] \\
\hline & Curcumin & 5 & 0.05 & 3.07 & $90 \%$ & - & - & - & [75] \\
\hline 28\%Zein:1\%Zein 4/1 & Ketoprofen & 10 & 58 & 2.91 & $5 \%$ & $100 \%$ & - & - & [79] \\
\hline PCL:Gelatin 1/2 & Metronidazole & 33.4 & 29 & -0.14 & $5 \%$ & $60 \%$ & $95 \%$ & $100 \%$ & [76] \\
\hline PCL:PVA 1/8 & $\begin{array}{l}\text { Metoclopramide } \\
\text { hydrochloride }\end{array}$ & 1 & $0.24^{\ddagger}$ & $2.36^{\ddagger}$ & $5 \%$ & $55 \%$ & $65 \%$ & $68 \%$ & [68] \\
\hline PLLA:PVA 1/8 & $\begin{array}{l}\text { Metoclopramide } \\
\text { hydrochloride }\end{array}$ & 1 & $0.24^{\ddagger}$ & $2.36^{\ddagger}$ & $2 \%$ & $12 \%$ & $22 \%$ & $25 \%$ & [68] \\
\hline PLGA:PVA $1 / 8$ & $\begin{array}{l}\text { Metoclopramide } \\
\text { hydrochloride }\end{array}$ & 1 & $0.24^{\ddagger}$ & $2.36^{\ddagger}$ & $5 \%$ & $38 \%$ & $62 \%$ & $72 \%$ & [68] \\
\hline PCL:PEG & salicylic acid & 10 & 1000 & 2.01 & $10 \%$ & $25 \%$ & $40 \%$ & - & [80] \\
\hline PEG:PLA $1 / 4$ & salicylic acid & 15 & 1000 & 2.01 & $0.1 \mathrm{mg} / \mathrm{mL}$ & $0.2 \mathrm{mg} / \mathrm{mL}$ & - & - & [70] \\
\hline $\begin{array}{l}\text { PEG:Cellulose Acetate } \\
\text { and Gelatin } 1 / 2\end{array}$ & amoxicillin & 3.7 & 0.58 & 0.88 & $22 \%$ & $100 \%$ & - & - & [73] \\
\hline PMMA:Nylon6 1/1 & ampicillin & 20 & 0.87 & 1.48 & $100 \mathrm{ug} / \mathrm{mL}$ & $300 \mathrm{ug} / \mathrm{mL}$ & $600 \mathrm{ug} / \mathrm{mL}$ & $800 \mathrm{ug} / \mathrm{mL}$ & [67] \\
\hline
\end{tabular}

${ }^{\dagger}$. Scifinder, Substance Identifier: Calculated using Advanced Chemistry Development (ACD/Labs) software v11.02; (accessed Jul 04, 2015).

f. DrugBank v4.3: Calculated using ALOGPS v2.1; (accessed Jul 04, 2015). 

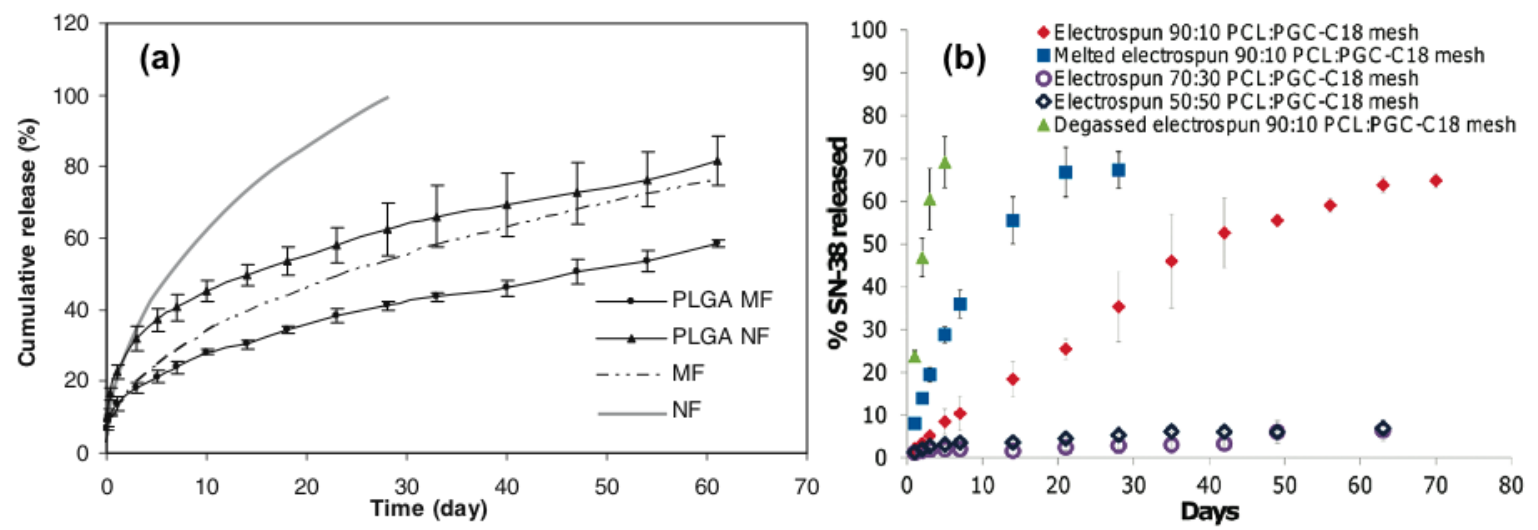

Figure 1. Examples of sustained release curves from electrospun uniaxial fibers: (a) $10 \mathrm{wt} \%$ loaded paclitaxel in PLGA microfibers (PLGA MF) and nanofibers (PLGA NF) and first order release curve fitting for microfibers (MF) $(k=0.119, n$ $=0.45)$ and nanofibers (NF) $(k=0.223, n=0.45)$. Release of paclitaxel sustained for 60 days to $80 \%$ cumulative release in PLGA NF and $60 \%$ cumulative release in PLGA MF. Fiber diameter significantly affected the release profile. [54]; (b) Release curves of SN-38, a bioactive agent, from blends of PCL and poly (glycerol monostearate-co- $\varepsilon$-caprolactone) (PGC-C18) at ratios of 90:10, 70:30, 50:50 PCL:PGC-C18. Effect of air displacement was minimal for the degassed 90:10 PCL:PGC-C18 mesh comparing to the native one. Melted mesh (at $80^{\circ} \mathrm{C}$ for $1 \mathrm{~min}$ ) reduced the pores and further eliminated time required for air displacement comparing to the native one. Higher PGC-C18 doping in the mesh significantly reduce the release rate. The effect on air displace has a direct influence on the wetting of the mesh and thus affects the release rate. [40] 
(a)

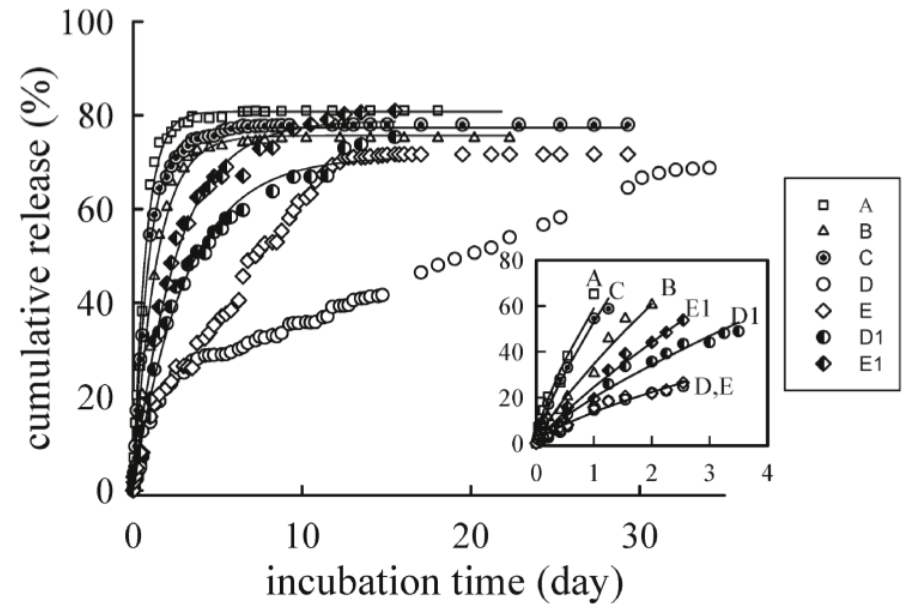

(b)

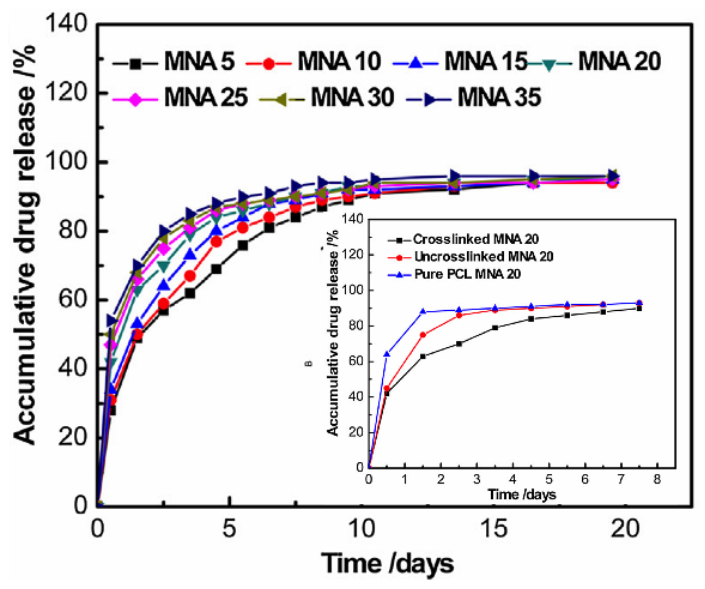

Figure 2. Examples of sustained release curves from electrospun coaxial fibers: (a) Cumulative release of DMOG from electrospun fibers. Figure legends: $A$ (PLA), B (PHB), C (PLA-core PHB-shell; Qc/Qs = 0.5/1.5; drug in shell), D (PLAcore PHB-shell; Qc/Qs = 0.5/1.5; drug in core), E (PLA-core PHB-shell; Qc/Qs = 2.0/1.5; drug in core), D1 (PHB-core PLA-shell; Qc/Qs = 1.5/0.5; drug in core), E1 (PHB-core PLA-shell; Qc/Qs = 1.5/2.0; drug in core). Fiber type D showed a sustained release of $70 \%$ of DMOG over 30 days. Switching core and shell polymers resulted in burst release due to different hydrophobicity of PLA and PHB. Shell thickness of fiber type $D$ is twice thicker than fiber type $E$ resulting in significant effect in release rate. [71]; (b) Cumulative release of metronidazole (MNA) loaded from $5 \mathrm{wt} \%$ to $35 \mathrm{wt} \%$ in the core of PCL-core gelatin-shell fibers (all gelatin shells were cross-linked). Higher drug loading increased the release rate. Insert showed the release curves on the effect gelatin shell crosslinking. Crosslinking of the gelatin shell resulted in changes of hydrophobicity and therefore changing the release behavior. [76] 


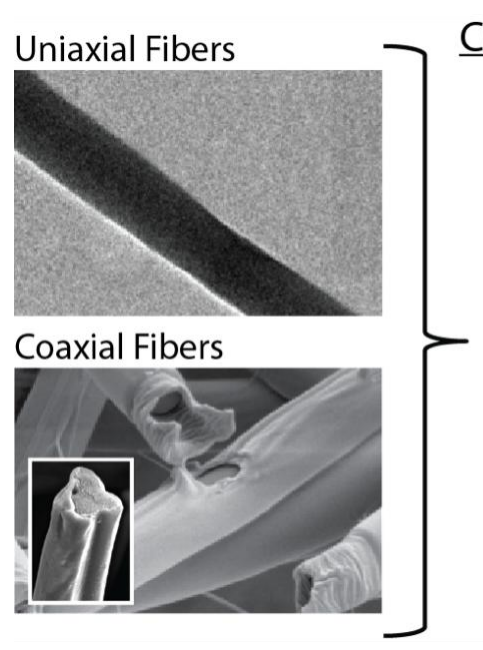

CONTROL VARIABLES

polymer Tg

polymer Tm

drug distribution solvent

drug crystallinity

fiber diameter core-shell diameter core-shell polymers drug properties/load etc...

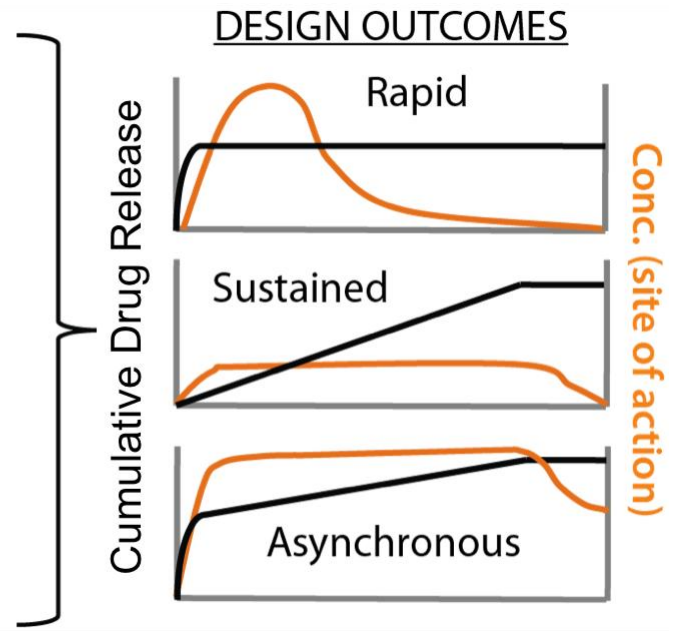

